\title{
Tourist Application for Selecting Packages: A Survey
}

\author{
Ameya Aniruddha Joshi \\ Department of Information \\ Technology \\ Trinity College of Engineering \\ and Research \\ Pune, India
}

\author{
Siddhant Sandeep Dube \\ Department of Information \\ Technology \\ Trinity College of Engineering \\ and Research \\ Pune, India
}

\author{
Omkar Ravindra Kadam \\ Department of Information \\ Technology \\ Trinity College of Engineering \\ and Research \\ Pune, India
}

\author{
Abhijit Manohar Shilamkar \\ Department of Information Technology \\ Trinity College of Engineering and Research \\ Pune, India
}

\author{
Gauri Bhagat \\ Department of Information Technology \\ Trinity College of Engineering and Research \\ Pune, India
}

\begin{abstract}
In today's world Travel and Tourism agencies are growing day by day as the Tourist's are increasing their part in traveling and visiting the different places. The main issue behind the this is the budget and the package selection in the travel and tourism industry because every agency think as if they are best compared to others, so to overcome such problems there must be a system that provides packages for the customer. The main objective of this project is to provide a suitable package to customer from different travel agencies by comparing packages provided by the agency's to the customer.
\end{abstract}

\section{Keywords}

Tourism, Travel, ARMA, NLP, Decision tree.

\section{INTRODUCTION}

Today, tourism is one of the biggest and dynamically developing sectors of economic activities. Its high expansion and growth rates, infrastructure amplification, and introduction of new management and educational experience actively acts various sectors of economy, which positively contribute to the social and economic development of the country as a whole. According to recent statistics, world's $10 \%$ income is coming from tourism and employs almost one tenth of the world's workforce.

Tourism's actual and potential economic impact is very jiggered. Throughout the world a major contributor to growth of economy is tourism industry. Tourism has created jobs in both large and small scales and is a major industry in many places. It is the dominant economic activity in some countries. The slogan 'Athithi Devo Bhava' which derives 'guests are like God' truly represents the fact that Indians have been highly hospitable people and this trend is still present in modern India. A guest is considered as highly honorable person. Going through a broader picture, the tourists visiting India are comprehensible. Foreigners love India as it is a warehouse for dance, culture, drama and art. Responsible tourism is conducted by way of giving the tourist the right concept in tours. Their accommodation in government lodges and private hotels is always a luxury. The importance to tourism is essential as they carry the picture of our sinology, culture and hospitality.

Several tourist spots do well throughout the year and in special seasons they perform exceptionally well in tourism as the foreign and domestic tourists throng the places for sightseeing or holiday trips like Goa. With the effective information and technological facilities, the stay of tourists has become more comfortable. Ministry of tourism is looking for new ways to encourage tourism in India like incredible India. It is very much essential for a tourist to enjoy the stay and is enhanced by facilities like road transport, guides and good hotels and delicious food. Many hotels and restaurants have multi-cuisines to accommodate the choices of foreign and domestic tourists. Tour organizers have become more concerned about tourist plans.

Complete details about weather conditions, clothes required and facilities are given to the tourists to help them prepare for a tour. This all has led to multi-dimensional changes in travel and tourism industry. This requirement starts by mitigating destination needs to the need for trained manpower for successful tourism management.

\section{LITERATURE SERVEY}

As we have discussed in the introduction about the tourism here are some papers on which have done Literature Survey.

Mirjana Peji'c Bach, Markus Schatten, Zrinka Maruši paper of "Data Mining Applications in Tourism" in September 2013 at Central European Conference on Information and Intelligent Systems they said

- These fields cover applications of data mining in forecasting, personalization, tourism management, tourism systems (such as recommendation systems), and machine learning.

- Keyword analysis comparing the two time periods was conducted.

- The first period revealed keywords oriented towards specific applications, such as segmentation, forecasting, and marketing.

- The second one revealed keywords oriented towards more advanced systems and technologies.

Yakun Hu, Dapeng Wu, Fellow, IEEE, and Antonio Nucci paper of "Fuzzy-Clustering-Based Decision Tree Approach for Large Population Speaker Identification " in April 2013 at IEEE transactions on audio, speech, and language processing said that

- Paper is all about the finding out noisy speakers under noisy conditions. 
- To mitigate this limitation, we propose a fuzzyclustering-based decision tree approach.

- Aims at using a hierarchical decision tree to partition the large population of all registered speakers into subgroups of very small population size and determining the speaker group at the leaf node to which a speaker under test belongs.

Enrico Avventi, Anders G. Lindquist, Life Fellow, IEEE, and Bo Wahlberg, Fellow's paper of "ARMA Identification of Graphical Models" in MAY 2013 at IEEE transactions on automatic control said that

- Paper is all about the representation of the components given to it.

- And it is processed through graphical representation (ARMA).

- We provide a step-by-step procedure to estimate the graphical structure and the corresponding ARMA model respecting the sparsity pattern. Some of these results are preliminary in nature, and further work is needed to test numerical algorithms and statistical procedures.

\section{CONCLUSION}

Our proposed system will be able to reduce the confusion and complexity the user faces in choosing packages and create a service accessible by all irrespective of region etc.

By rendering the service to users through a website format can greatly have an impact on the system's mass reach. We are going to search for relevant data and find out the patterns in them. On the basis of traveler's historical data, we generate a result while offering packages for suggesting place to visit.

Traveler's data can be gathered and analyzed using ARMA and Machine learning's ID3 algorithm with NLP. The Travel history is then analyzed using ARMA to predict user's behaviour and future choices on a web based platform and by implementing a cloud based database storage.

As a scope for the future we can use this project to be implemented on a large scale i.e. for large number of users. Can later be transported to other devices such as Smart Mobile Phones, Tablets and Kiosk Stations and can also be implemented on a large business perspective scale.

\section{ACKNOWLEDGMENTS}

The completion of this phase of this project is due to support, guidance, help, hard work, motivation, so it is our pleasure to represent the idea of "Web application that selects tourist places and packages for the traveler and provides the best offers by comparisons from different agencies using NLP, Machine learning, ARMA". So we are thankful firstly to the Principal of Trinity College of engineering Prof. P.S. Dabeer and HOD (Head of Department) ma'am Prof.S.E. Pawar and our Project Guide Prof. Gauri Bhagat for support and guidance. Also we thankful to the staff of Information Technology department for helping us.

\section{REFERENCES}

[1] Mirjana Pejic Bach, Markus Schatten, Zrinka Marui,Data Mining Applicationsin Tourism, at Central European Conference on Information and Intelligent Sys-tems, on September 2013.

[2] Erik Cambria, Bjoirn Schuller, Yunqing Xia, Catherine Havasi, New Avenues inOpinion Mining and Sentiment Analysis at IEEE Computer Society on March2013.

[3] Yakun Hu, Dapeng Wu, Fellow, IEEE, and Antonio Nucci, Fuzzy-Clustering-Based Decision Tree Approach for Large Population Speaker Identi_-cation , at IEEE TRANSACTIONS ON AUDIO, SPEECH, AND LANGUAGEPROCESSING, on April 2013, VOL. 21, NO. 4.

[4] Enrico Avventi, Anders G. Lindquist, Life Fellow, ARMA Identi_cation ofGraphical Models, at IEEE, and Bo Wahlberg, Fellow, IEEE TRANSACTIONSON AUTOMATIC CONTROL, on MAY 2013, VOL. 58, NO. 5 .

[5] Pairaya J, Buddhagarn R, Sukree S and Punthumadee K, An Innovation of Travel Information Gathering Framework, at World Academy of Science, En-gineering and Technology ,on 2012.

[6] Pairaya Juwattanasamran, Sarawut Supattranuwong, Sukree Sinthupinyo, Apply-ing Data Mining to Analyze Travel Pattern in Searching Travel Destina-tion Choices , at The International Journal Of Engineering And Science (IJES)on 2013.

[7] Stanley Loh,Fabiana Lorenzi,Ramiro Saldana,Daniel Licthnow, A Tourism rec-ommender system based on collaboration and text analysis, at The Infor-mation Technology Tourism, Vol. 6 ,Copyright 2004 Cognizant Comm. Corp.

[8] Tzu-Ching Lin, Enhancing Tourism Intermediaries with the Data MiningProcess TransWorld University Taiwan,2012 International Conference on Informa-tion and Knowledge Management.

[9] Markus Laner, Philipp Svoboda, Markus Rupp, Parsimonious Network Tra_cModeling By Transformed ARMA Models, at The (IEEE Access),on Decem-ber 17, 2013.

[10] Leszek Rutkowski, Maciej Jaworski, Lena Pietruczuk, Piotr Duda, Decision Treesfor Mining Data Streams Based on the Gaussian Approximation,at TheIEEE TRANSACTIONS ON KNOWLEDGE AND DATA ENGINEERING, VOL.26, NO. 1, JANUARY 2014.

[11] Ralph Grishman, Information Extraction in Natural Language Process-ing,at The New York University for IEEE Computer Society,on March 2014.

[12] Helmut Bergerv, Michael Dittenbach, Dieter Merkl, Anton Bogdanovych, SimeonSimo_ and Carles Sierra, Opening new dimensions for e-Tourism, at TheSpringerVerlag London Limited 2006,on 8th December 2006. 